\title{
Research of rheological properties of fermented milk ice cream with high content of exopolysaccharides
}

\author{
Ekaterina Pozhidaeva ${ }^{1 *}$, Lyubov Golubeva ${ }^{1}$, Anton Sadchenko ${ }^{2}$, and Yana Dymovskih ${ }^{1}$ \\ ${ }^{1}$ Department of Technology of Animal ProductsVoronezh State University of Engineering Technologies, Voronezh, Russia \\ ${ }^{2}$ Short stay hospital, SBHI "SCH of S.I. Spasokukotsky MHD", Moscow, Russia
}

\begin{abstract}
As a result of scientific and technological research, the ingredient composition and the feasibility of using complex bacterial starter cultures as part of fermented milk ice cream have been substantiated. The objects of the study were samples of mixtures for the production of fermented milk ice cream were considered, which included dairy and non-dairy components, including complex bacterial starter cultures: YF-L812 (Streptococcus thermophilus, Lactobacillus bulgaricus) - control sample and YO-PROX 777 (Streptococcus thermophilus, Lactobacillus delbrueckii ssp.bulgaricus), as well as pectin stabilizers "Grindsted Pektin LC 710" and "Cremodan SE 334" with a concentration of 0.1-0.7\%. It was found that a prototype sample with starter culture YO-PROX 777 has increased values of dynamic viscosity compared with the control sample with similar stabilizers and their identical concentrations, which is evidence of the accumulation of exopolysaccharides in the fermented fermented milk mixture. A prototype of fermented milk ice cream has better shape stability during temperature control, the thawing rate is reduced by 1.6 times compared to the control. The degree of overrun of the prototype fermented milk ice cream is $37.6 \%$, which is 1.2 times higher compared to the control.
\end{abstract}

\section{Introduction}

Expanding the range of new products that have a positive effect on the human microbiome is an urgent task dictated by the current state of the consumer market. In addition, there is currently an increase in demand for low-calorie and sugar-free products, which is due to the disappointing increasing dynamics of the population sickness of the Russian Federation [1, 2]. A special place in the assortment of such food systems is occupied by fermented milk ice cream, which allows maintaining viable cells for a long time, which significantly increases their logistic potential. However, during the freezing process, the structure of the product may be destroyed due to changes in the moisturebinding ability of the ingredients that make up the product $[3,4]$.

An analysis of domestic and foreign experience in the production of milk-based products, in particular fermented milk ice cream, indicates almost complete absence of exopolysaccharides in the composition, which are the main metabolites of microflora and perform a number of important functions: biological in relation to microorganisms, biocorrective in relation to macroorganism and technological in relation to food systems. Exopolysaccharides have a high moisturebinding ability, they form a thick, stretching consistency $[5,6]$. As a result of the synthesis of microbial exopolysaccharides, they accumulate in the fermentable system, which is accompanied by additional moisture binding. By changing the state of moisture in fermented systems, which also leads to a change in structural and mechanical characteristics, conclusions can be drawn on the quantitative characteristics of the biosynthesis of exopolysaccharides $[7,8]$.

The aim of the work is to design the component composition of fermented milk ice cream with a high content of exopolysaccharides and improved rheological characteristics.

\section{Experimental}

As objects of research, samples of mixtures for the production of fermented milk ice cream were considered, which included dairy and non-dairy components, including complex bacterial starter cultures: YF-L812 (Streptococcus thermophilus, Lactobacillus bulgaricus) - control sample and YOPROX 777 (Streptococcus thermophilus, Lactobacillus delbrueckii ssp.bulgaricus), as well as pectin stabilizers "Grindsted Pektin LC 710" and "Cremodan SE 334" with a concentration of $0.1-0.7 \%$. The dynamic viscosity of the experimental and control samples was evaluated using a Brookfield RV viscometer with varying rotational speeds in the range of $10-100 \mathrm{rpm}$.

In the studied fermented milk ice cream samples, shape stability was determined, characterizing their ability to maintain geometric dimensions at a temperature of $20 \pm 1{ }^{\circ} \mathrm{C}$. The studies were carried out by taking a cylindrical sample with a diameter of 36 $\mathrm{mm}$, a height of $4-5 \mathrm{~cm}$ and placing the sample on a pre-cooled Petri dish. The prepared sample of fermented milk ice cream was placed in a thermostat with an air temperature of $20 \pm 1{ }^{\circ} \mathrm{C}$ with further fixation of the area of the sample at regular intervals until the 
sample lost its geometric dimensions. The overrun degree of fermented milk ice cream was determined by the mass difference before and after freezing.

\section{Results and discussion}

It was found that with an increase in the concentration of stabilizers "Grindsted Pektin LC 710" and "Cremodan SE 334" from 0.1 to $0.7 \%$ at a spindle speed of $10 \mathrm{rpm}$ and a torque of $0.2-0.4 \mathrm{~N}$ in the test sample of fermented milk ice cream containing starter culture YOPROX 777, values $\mu$ of dynamic viscosity increase from 11.6 to 35.3 and from 14.5 to $19.8 \mathrm{MPa} \cdot \mathrm{s}$ (Fig. 1, 2). In the control sample containing a starter culture YF-L812, the same dynamic viscosity values increased from 10.4 to 20.2 and from 12.1 to $29.5 \mathrm{MPa} \cdot \mathrm{s}$. A further increase in the spindle speed to $100 \mathrm{rpm}(4.0-6.5 \mathrm{~N} \cdot \mathrm{m})$ leads to an increase in the same values $\mu$ of dynamic viscosity in the ranges: $37.6-70.9$ and $48.3-58.5$, as well as 51.471.1 and 52.6-74.7 $\mathrm{MPa} \cdot \mathrm{s}$, respectively [8]. The data obtained indicate increased values $\mu$ of dynamic viscosity in the test sample with starter culture YOPROX 777, compared with the control sample with similar stabilizers and their identical concentrations, which is evidence of the accumulation of exopolysaccharides in the fermented fermented milk mixture [9].

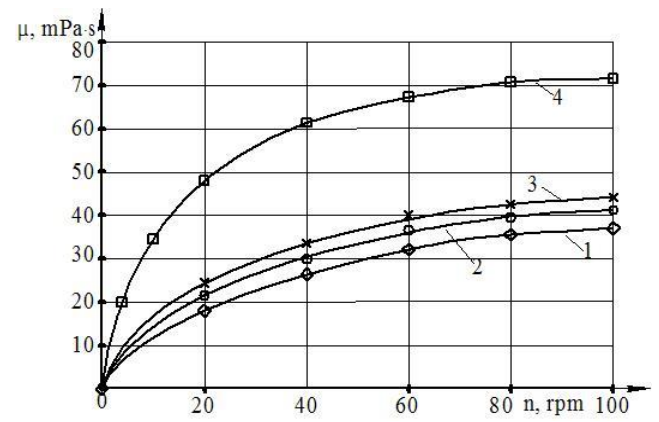

a

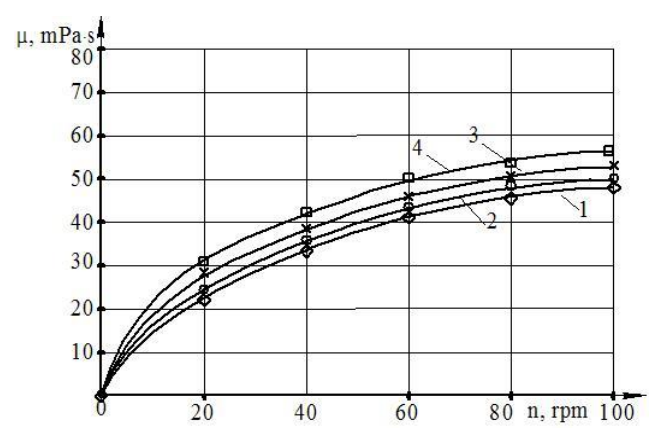

b

Fig. 1. The dependence of dynamic viscosity on the shear rate gradient of a mixture for fermented milk ice cream containing starter culture YO-PROX 777 at various concentrations of stabilizers: a - "Grindsted Pektin LC 710", b - "Cremodan SE 334": 1 - 0,1\%, 2 - 0,3\%, 3-0,5\%, 4 $0,7 \%$

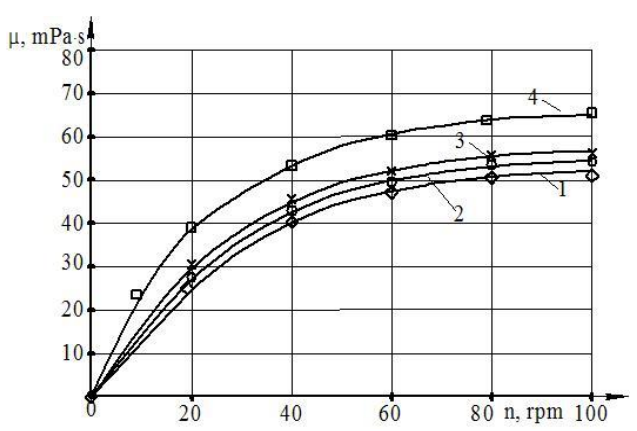

a

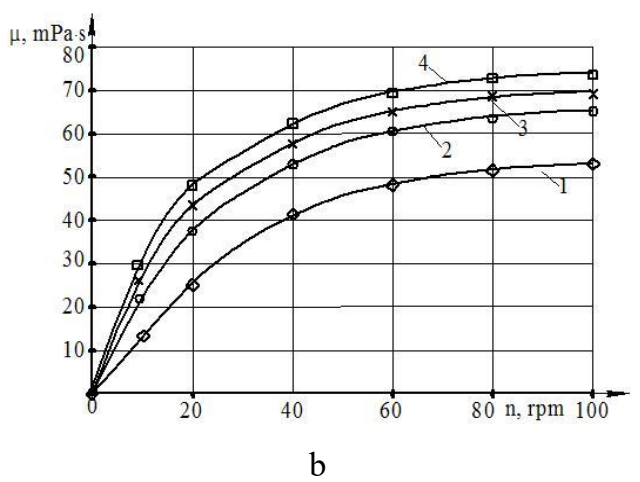

Fig. 2. The dependence of dynamic viscosity on the shear rate gradient of a mixture for fermented milk ice cream containing a starter culture YF-L812 at various concentrations of stabilizers: a - "Grindsted Pektin LC 710", b - "Cremodan SE 334": 1 - 0,1\%, 2 - 0,3 \%, 3 - 0,5\%, 4 $0,7 \%$

An important indicator of the quality of ice cream, both for the producer and for the consumer, is its resistance to thawing and shape retention $[10,11]$. It has been established that the fermented milk ice cream with the addition of the starter culture YF-L812, which completely melted after 35 minutes, has the least formstability. The sample with the introduction of starter culture YO-PROX 777 has more pronounced structural and mechanical properties, which is due to the presence of exopolysaccharides, and the best indicators of shape stability during temperature control. The prototype partially melted, fermented milk ice cream was characterized by a homogeneous structure. The data obtained make it possible to calculate and predict the rate of thawing of fermented milk ice cream samples, which varies depending on the qualitative composition. In the prototype the melting rate decreased by 1.6 times compared with the control.

The data obtained indicate an increase in the degree of moisture binding in the experimental sample, which presumably contributes to the formation of smaller ice crystals during freezing and to improve the structural and mechanical characteristics of desserts. This circumstance allows us to predict and directionally form not only the structural and mechanical characteristics of the final food products, but also helps to increase the shelf life due to a decrease in the agglomeration rate and the size of ice crystals.

One of the indicators of the quality of ice cream is its overrun degree. The overrun degree of fermented milk ice cream with the introduction of starter culture YO-PROX 777 is $37.6 \%$, in the control sample $-32.4 \%$, 
which is due to the increased content of exopolysaccharides in the experimental sample.

It was experimentally established that the number of fermented milk microorganisms in the experimental samples during storage for 6 months remained at the level of at least $10^{7} \mathrm{CFU} / \mathrm{g}$ of the product, which indicates their high eubiotic activity [12]

\section{Conclusion}

As a result of scientific and technological studies, the feasibility of using yeast starter culture YO-PROX 777, which provides an increased content of exopolysaccharides in the finished product, is substantiated. The data obtained indicate increased values of dynamic viscosity in the test sample with starter culture YO-PROX 777, compared with the control sample with similar stabilizers and their identical concentrations. The quantitative characteristics of the form stability level increase and the overrun degree of new fermented milk ice cream were established, the thawing rate decreased by 1.6 times, and the overrun degree increased by 1.2 times compared to the control. The high concentration stability of lactic fermented microorganisms during storage for up to 6 months was revealed, which objectively testifies the increased consumer characteristics of ice cream and its functional properties.

\section{References}

1. N. Konar, I. Palabiyik, O. SaidToker, D.

GencPolat, E. Kelleci, H. Rasouli Pirouzian, A.

Akcicek, and O. Sagdic, Journal of Functional

Foods, 43, 206-213 (2018)

2. Nurul FarhanaFazilah, Arbakariya B. Ariff, Mohd

EzuanKhayat, LeonardoRios-Solis, and

MurniHalim, Journal of Functional Foods, 48,

387-399 (2018)
3. Carolina Iraporda, Irene A. Rubel, Guillermo D. Manrique, and G. Analía, LWT, 101, 738-746 (2019)

4. A.A. Tvorogova, Empire of Cold, 2, 70-72 2018.

5. S.I. Artyukhova and E.V. Motornaya, International Journal of Experimental Education, 5-1, 76 (2015)

6. V.I. Ganina, News of universities. Food technology, 5-6, 65-66 (2005)

7. E.A. Pozhidaeva, E.S. Popov, A.V. Ilyushina, N.V. Bolotova, and E.V. Ivanova, Food Industry, 11, 73-77 (2018)

8. L.V. Golubeva, A.A. Smirnykh, and E.A.Pozhidaeva, Bulletin of the International Academy of Refrigeration, 4, 45-47 (2009)

9. N.S. Rodionova, A.A. Rodionov, E.S. Popov, and T.A. Razinkova, News of Universities. Applied Chemistry and Biotechnology, 8, 4, 95-105 (2018)

10. D.S. Mileeva and A.A. Tvorogova, World of ice cream and quick-frozen foods, 1, 22-24 (2019)

11. N.S. Rodionova, K.K. Polyansky, and E.S. Popov, Dairy industry, 2, 58-60 (2020)

12. E.A. Pozhidaeva, M.A. Shvyreva, and Y.A. Dymovskikh, Actual biotechnology, 2, 263 (2017) 